\title{
Bifurcation Analysis of an SIR Epidemic Model with the Contact Transmission Function
}

\author{
Guihua $\mathrm{Li}^{1}$ and Gaofeng $\mathrm{Li}^{2}$ \\ ${ }^{1}$ School of Science, North University of China, Taiyuan, Shanxi 030051, China \\ ${ }^{2}$ Xinjiang Agriculture Second Division Korla Hospital, Korla, Xinjiang 841000, China
}

Correspondence should be addressed to Guihua Li; ttl1013@163.com

Received 8 December 2013; Accepted 23 December 2013; Published 21 January 2014

Academic Editor: Kaifa Wang

Copyright (C) 2014 G. Li and G. Li. This is an open access article distributed under the Creative Commons Attribution License, which permits unrestricted use, distribution, and reproduction in any medium, provided the original work is properly cited.

\begin{abstract}
We consider an SIR endemic model in which the contact transmission function is related to the number of infected population. By theoretical analysis, it is shown that the model exhibits the bistability and undergoes saddle-node bifurcation, the Hopf bifurcation, and the Bogdanov-Takens bifurcation. Furthermore, we find that the threshold value of disease spreading will be increased, when the half-saturation coefficient is more than zero, which means that it is an effective intervention policy adopted for disease spreading. However, when the endemic equilibria exist, we find that the disease can be controlled as long as we let the initial values lie in the certain range by intervention policy. This will provide a theoretical basis for the prevention and control of disease.
\end{abstract}

\section{Introduction}

The classical SIR model for disease transmission has been widely studied. It is one of the most important issues that the dynamical behaviors are changed by the different incidence rate in epidemic system. For the incidence rate, we divided into two categories: one is that Capasso and Serio [1] proposed the infection force which is a saturated curve, described "crowding effect" or "protection measures;" the other is the infection force that describes the effect of "intervention policy," for example, closing schools and restaurants and postponing conferences (see Figure 1). For the model with the saturated infection force, $a I^{2} /\left(b+I^{2}\right)$, which is one of the typical infection forces, the rich dynamical behaviors were founded by Ruan and Wang [2] and Tang et al. [3]. The model with the incidence rate can be suited for many infectious diseases, including measles, mumps, rubella, chickenpox, and influenza. For more research literatures about nonlinear infection rate see [4-9]. However, for some parasite-host models, by observing macro- and microparasitic infections, one finds that the infection rate is an increasing function of the parasite dose, usually sigmoidal in shape $[10,11]$. So we will build a model with sigmoidal incidence rate which is taken into account "crowding effect" and "saturated effect."
According to the parasite-host model which is proposed by Anderson and May $(1979)[12,13]$, the model is as follows:

$$
\begin{aligned}
& \frac{d S}{d t}=A-d S-\beta(I) S, \\
& \frac{d I}{d t}=\beta(I) S-(d+\gamma+\epsilon) I, \\
& \frac{d R}{d t}=\gamma I-d R,
\end{aligned}
$$

where $S, I, R$ are susceptible hosts, infected hosts, and removed hosts, respectively. $A$ is the birth rate of susceptible host, $d$ is the natural death rate of a population, $\gamma$ is the removal rate, and $\epsilon$ is the per capita infection-related death rate. If we denote infection force $\beta(I)=g(I) I, g(I)$ can be explained as the rate of valid contact. At the beginning of disease, most people have poor awareness of prevention, then the rate of valid contact $g(I)$ can be first increasing then tends to a certain value. As the time flies, people are gradually aware of the seriousness and take measures to prevent and control development of the disease and will reduce to be contact with infected, so the rate of contact $g(I)$ is first increasing then 


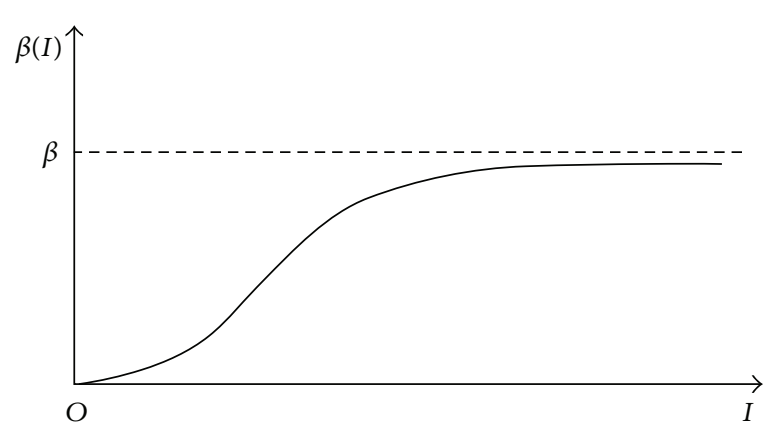

(a) Case $0 \leq a$

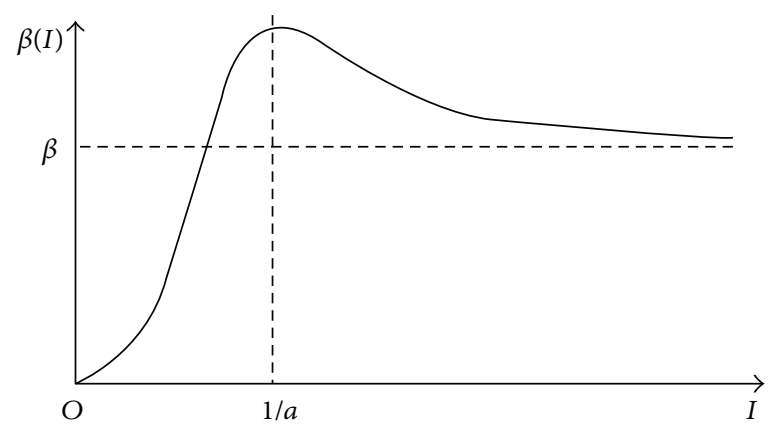

(b) Case $-2 \sqrt{b}<a<0$

FIGURE 1: The plotting for the contact transmission function $\beta(I)$.

decreasing. In short time, it does not tend to zero, but tends to a nonzero constant. To simplify the study, we take

$$
g(I)=\frac{\beta I^{2}}{b+a I+I^{2}},
$$

where $b>0$ and $-2 \sqrt{b}<a$. If $a \geq 0, g(I)$ is increasing monotonically and tends to $\beta$. If $-2 \sqrt{b}<a<0, g(I)$ is first increasing then decreasing and tends to $\beta$ (see Figure 1 ).

Then model (1) becomes

$$
\begin{aligned}
& \frac{d S}{d t}=A-d S-\frac{\beta S I^{3}}{b+a I+I^{2}}, \\
& \frac{d I}{d t}=\frac{\beta S I^{3}}{b+a I+I^{2}}-(d+\gamma+\epsilon) I, \\
& \frac{d R}{d t}=\gamma I-d R,
\end{aligned}
$$

where $\beta$ is the valid contact coefficient.

When $a=0$ and $b=0$, model (3) becomes

$$
\begin{gathered}
\frac{d S}{d t}=A-d S-\beta S I, \\
\frac{d I}{d t}=\beta S I-\mu I, \\
\frac{d R}{d t}=\gamma I-d R,
\end{gathered}
$$

where $\mu=d+\gamma+\epsilon$.

We know that $R_{0}=\beta A / d \mu$ is the basic reproduction number of (4). It is easy to see that there is a unique positive equilibrium $I^{*}$ in system (4) when $R_{0}>1$ and there is no positive equilibrium when $R_{0} \leq 1$. In the next sections, we will study that parameters $a$ and $b$ would have any effect on the dynamic behaviors of model (3).

The organization of this paper is as follows. In the next section, we analyze the existence and stability of the endemic equilibria for model (3). Then we discuss conditions for the Hopf bifurcation and the Bogdanov-Takens bifurcation in Sections 3. Section 4 presents numerical simulations to indicate dynamical behaviors and bifurcation structures, and gives with a brief discussion.

\section{Existence and Stability of Equilibria}

We consider the positive equilibria of (3). Setting the right hand sides of system (3) to zero, we find that the first and second equations of system (3) do not include $R$, so we only consider

$$
\begin{gathered}
A-d S-\frac{\beta S I^{3}}{b+a I+I^{2}}=0, \\
\frac{\beta S I^{3}}{b+a I+I^{2}}-\mu I=0 .
\end{gathered}
$$

From the above two equations, except for the disease-free equilibrium (DFE) at $(A / d, 0)$, any endemic equilibrium (EE), if exists, is the intersection of the following two curves in the positive quadrant

$$
\begin{aligned}
& S=\frac{A-\mu I}{d}, \\
& S=\frac{\mu\left(b+a I+I^{2}\right)}{\beta I^{2}} .
\end{aligned}
$$

From (6), I must satisfy the following equation:

$$
H(I):=\beta I^{3}+d\left(1-R_{0}\right) I^{2}+d a I+d b=0 .
$$

Thus the intersection of two curves (6) is transformed into the positive root of (7).

The derivative of $H^{\prime}(I)$ is

$$
H^{\prime}(I):=3 \beta I^{2}+2 d\left(1-R_{0}\right) I+d a .
$$

In the following, we consider three cases according to the sign of $a$. By calculation, we have the following three theorems.

Set

$$
\begin{gathered}
a_{1}=d^{2}\left(1-R_{0}\right)^{2}-3 \beta a d, \\
a_{2}=27 \beta^{2} d b-d b\left(1-R_{0}\right)\left(3 \beta a d-2 a_{1}\right) .
\end{gathered}
$$

Theorem 1. Suppose $a>0$. Then we have the following.

(a) If $R_{0} \leq 1+\sqrt{3 \beta a / d}$, then system (3) has no endemic equilibrium. 
(b) If $R_{0}>1+\sqrt{3 \beta a / d}$, then we have the following.

(i) When $2 a_{1}^{3 / 2}<a_{2}$, system (3) has no endemic equilibrium.

(ii) When $2 a_{1}^{3 / 2}=a_{2}$, system (3) has a unique endemic equilibrium.

(iii) When $2 a_{1}^{3 / 2}>a_{2}$, system (3) has two endemic equilibria $E_{1}\left(S_{1}, I_{1}\right), E_{2}\left(S_{2}, I_{2}\right)$.

Theorem 2. Suppose $a=0$. Then we have the following.

(a) If $R_{0}<1+\sqrt[3]{27 \beta^{2} b / 4 d^{2}}$, then system (3) has no endemic equilibrium.

(b) If $R_{0}=1+\sqrt[3]{27 \beta^{2} b / 4 d^{2}}$, then system (3) has a unique endemic equilibrium.

(c) If $R_{0}>1+\sqrt[3]{27 \beta^{2} b / 4 d^{2}}$, then system (3) has two endemic equilibria $E_{1}\left(S_{1}, I_{1}\right), E_{2}\left(S_{2}, I_{2}\right)$.

Theorem 3. Suppose $-2 \sqrt{b}<a<0$. Then we have the following.

(a) If $2 a_{1}^{3 / 2}<a_{2}$, then system (3) has no endemic equilibrium.

(b) If $2 a_{1}^{3 / 2}=a_{2}$, then system (3) has a unique endemic equilibrium $E^{*}\left(S^{*}, I^{*}\right)$.

(c) If $2 a_{1}^{3 / 2}>a_{2}$, then system (3) has two endemic equilibria $E_{1}\left(S_{1}, I_{1}\right), E_{2}\left(S_{2}, I_{2}\right)$, where $I_{1}<I^{*}<I_{2}$.

Remark 4. From Theorems 1 and 2, we can find that the basic reproduction number for the model (3) is less than that of the standard model. It means that the disease will spread more easily. For Theorem 3, it is obvious that the disease can exist if $R_{0}<1$.

For disease-free equilibrium (DFE), it is easy to calculate that the Jacobian matrix of system (3) at DFE has eigenvalues $\lambda_{1}=-d$ and $\lambda_{2}=-\mu$. Hence, DFE is always stable.

In the following, the stability of the endemic equilibrium in system (3) will be studied. Firstly, evaluating the Jacobian matrix of system (3) at $E(S, I)$ gives

$$
J=\left.\left(\begin{array}{ll}
j_{11} & j_{12} \\
j_{21} & j_{22}
\end{array}\right)\right|_{(S, I)},
$$

where

$$
\begin{array}{cc}
j_{11}=-d-\frac{\beta I^{3}}{b+a I+I^{2}}, & j_{12}=-\mu-\frac{\mu(2 b+a I)}{b+a I+I^{2}}, \\
j_{21}=\frac{\beta I^{3}}{b+a I+I^{2}}, & j_{22}=\frac{\mu(2 b+a I)}{b+a I+I^{2}} .
\end{array}
$$

Its characteristic equation is

$$
P(\lambda)=\lambda^{2}-\operatorname{tr}(J) \lambda+\operatorname{det}(J)=0,
$$

where

$$
\begin{aligned}
& \operatorname{det}(J)=\frac{\beta \mu I^{3}-d a \mu I-2 d b \mu}{b+a I+I^{2}}, \\
& \operatorname{tr}(J)=\frac{-\beta A I^{2}+a \mu^{2} I+2 b \mu^{2}}{\mu\left(b+a I+I^{2}\right)} .
\end{aligned}
$$

It is easy to calculate

$$
\beta \mu I^{* 3}-d a \mu I^{*}-2 d b \mu=\mu\left[I^{*} H^{\prime}\left(I^{*}\right)-2 H\left(I^{*}\right)\right]=0 ;
$$

that is, $\left.\operatorname{det}(J)\right|_{I=I^{*}}=0$.

Now suppose that the model has two endemic equilibria $E_{1}\left(S_{1}, I_{1}\right), E_{2}\left(S_{2}, I_{2}\right)$, with $I_{1}<I^{*}<I_{2}<A / \mu$; that is, in Theorem 1, the item (b) (iii) holds or Theorems 2 and 3, the item $c$ holds. If $J_{i}(i=1,2)$ is the Jacobian matrix at $\left(S_{i}, I_{i}\right)$, then (13) gives

$$
\operatorname{det}\left(J_{i}\right)=\left.\frac{\beta \mu I^{3}-d a \mu I-2 d b \mu}{b+a I+I^{2}}\right|_{I=I_{i}} .
$$

Thus, it is easily obtained that $\operatorname{det}\left(J_{1}\right)$ is negative and $\operatorname{det}\left(J_{2}\right)$ is positive. We can immediately conclude that the endemic equilibrium $E_{1}$ with low number of infected individuals is always a saddle, and that the endemic equilibrium $E_{2}$ with high number of infected individuals is a node or focus but the stability of $E_{2}$ is determined by $\operatorname{tr}\left(J_{2}\right)$. From (14), we notice that the sign of the trace of $J_{2}$ is determined by

$$
\operatorname{tr} 1:=-\beta A I^{2}+a \mu^{2} I+2 b \mu^{2} .
$$

Set

$$
\begin{gathered}
b_{0}:=\frac{a \mu^{2}+\mu \sqrt{a^{2} \mu^{2}+8 \beta A b}}{2 \beta A}, \\
b_{1}:=\frac{-d\left(1-R_{0}\right)+\sqrt{a_{1}}}{3 \beta \mu}, \\
r_{0}:=\beta A\left(a b_{0}(A+1)+A b\right), \\
r_{1}:=d\left(R_{0}-1\right)(2+A)\left(a+2 b_{0}\left(1-R_{0}\right)\right) .
\end{gathered}
$$

Theorem 5. Assume that (3) has two endemic equilibria. Then $E_{2}$ is asymptotically stable if one of the following is satisfied.

(a) $b_{0}<b_{1}$

(b) $b_{0}>b_{1}$ and $r_{0}>r_{1}$.

Further, $E_{2}$ is unstable if $b_{0}>b_{1}$ and $r_{0}<r_{1}$.

Proof. If $b_{0}<b_{1}$, then $-\beta A I^{* 2}+a \mu^{2} I^{*}+2 b \mu^{2}<0$. It follows from $I_{2}>I^{*}$ that $\operatorname{tr} 1<0$. Hence, $E_{2}$ is asymptotically stable in this case. If $b_{0}>b_{1}$, we have $b_{0}>I^{*}$. By direct calculations we see that $r_{0}>r_{1}$ implies $I_{2}>b_{0}$, which leads to tr $1<0$. Therefore, $E_{2}$ is asymptotically stable if condition (b) holds. Similarly, if $b_{0}<b_{1}$ and $r_{0}<r_{1}$, we have $I_{2}>b_{0}$, which leads to $\operatorname{tr} 1>0$. It follows that $E_{2}$ is unstable. 


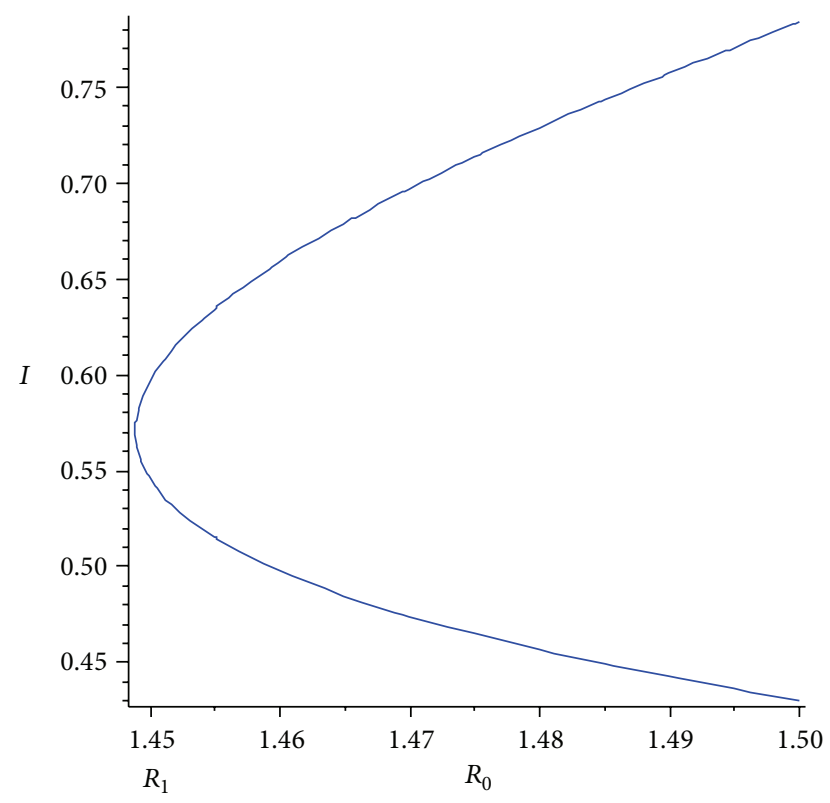

FIgURE 2: The bifurcation curves the palne of $\left(I, R_{0}\right)$.

\section{Bifurcation of the System}

3.1. Hopf Bifurcation. When the condition (b) (ii) in Theorem 1 and the condition (b) in Theorems 2 and 3 hold and $r_{0}=$ $r_{1}$, there are a pair of purely imaginary eigenvalues (Figure 2). Thus for suitable parameter values a Hopf bifurcation may occur, which means that there is a periodic solution around the larger nontrivial equilibrium. In order to determine the type of the Hopf bifurcation, we set

$$
\begin{gathered}
q_{1}:=\frac{\rho p^{3}\left(3 \beta b A+a^{2}-4 b\right)}{(2 b \mu p+a \rho A)^{2}}, \\
q_{2}:=\frac{-2 \beta S_{2}\left[\left(a^{2}-b\right) b I_{2}^{4}+4 a b I_{2}^{3}+6 b^{2} I_{2}^{2}-b^{3}\right]}{\left(b+a I_{2}+I_{2}\right)^{4}} .
\end{gathered}
$$

Then we consider the transformation $X=S-S_{2}, Y=I-I_{2}$ to move $\left(S_{2}, I_{2}\right)$ to the origin of $(X, Y)$. After some manipulations, the model can be transformed into the following equations:

$$
\begin{aligned}
& \frac{d X}{d t}=a_{11} X+a_{12} Y-C(X, Y), \\
& \frac{d Y}{d t}=b_{11} X+b_{12} Y+C(X, Y),
\end{aligned}
$$

where $C(X, Y)$ represents the higher order terms and

$$
\begin{array}{cc}
a_{11}=-d-\frac{\beta I_{2}^{3}}{b+a I_{2}+I_{2}^{2}}, & a_{12}=-\mu-\frac{\mu\left(2 b+a I_{2}\right)}{b+a I_{2}+I_{2}^{2}}, \\
b_{11}=\frac{\beta I_{2}^{3}}{b+a I_{2}+I_{2}^{2}}, & b_{12}=\frac{\mu\left(2 b+a I_{2}\right)}{b+a I_{2}+I_{2}^{2}} .
\end{array}
$$

Suppose $r_{0}=r_{1}$. Then

$$
\operatorname{tr}(J)=-d-\frac{\beta I_{2}^{3}}{b+a I_{2}+I_{2}^{2}}+\frac{\mu\left(2 b+a I_{2}\right)}{b+a I_{2}+I_{2}^{2}}=0 .
$$

By defining $\rho=\beta I_{2}^{3} /\left(b+a I_{2}+I_{2}^{2}\right)$ and $p=\mu\left(2 b+a I_{2}\right) /(b+$ $a I_{2}+I_{2}^{2}$ ), it can be seen that

$$
\begin{gathered}
a_{11}=-d-\rho, \quad a_{12}=-\mu-p, \quad b_{11}=\rho, \\
b_{12}=p, \quad d=p-\rho .
\end{gathered}
$$

Set

$$
\omega=\sqrt{\operatorname{det}\left(J_{2}\right)}=\sqrt{\mu \rho-d p}
$$

Then the eigenvalues of $J_{2}$ are $\lambda_{1}=\omega i$ and $\lambda_{2}=-\omega i$.

Now, using the transformation $u=X, v=-(1 / \omega)\left(a_{11} X+\right.$ $\left.a_{12} Y\right)$ to (20), we obtain

$$
\begin{aligned}
& \frac{d u}{d t}=-\omega v+F_{1}(u, v), \\
& \frac{d v}{d t}=\omega u+F_{2}(u, v),
\end{aligned}
$$

where

$$
\begin{aligned}
& F_{1}(u, v)=-C\left(u, \frac{\omega v-p u}{\mu+p}\right), \\
& F_{2}(u, v)=\frac{\mu}{\omega} C\left(u, \frac{\omega v-p u}{\mu+p}\right) .
\end{aligned}
$$

If

$$
\begin{gathered}
\sigma=\frac{1}{16}\left[\frac{\partial^{3} F_{1}}{\partial u^{3}}+\frac{\partial^{3} F_{2}}{\partial u \partial v^{2}}+\frac{\partial^{3} F_{2}}{\partial u^{2} \partial v}+\frac{\partial^{3} F_{2}}{\partial v^{3}}\right] \\
+\frac{1}{16 \omega}\left[\frac{\partial^{2} F_{1}}{\partial u \partial v}\left(\frac{\partial^{2} F_{1}}{\partial u^{2}}+\frac{\partial^{2} F_{1}}{\partial v^{2}}\right)-\frac{\partial^{2} F_{2}}{\partial u \partial v}\left(\frac{\partial^{2} F_{2}}{\partial u^{2}}+\frac{\partial^{2} F_{2}}{\partial v^{2}}\right)\right. \\
\left.-\frac{\partial^{2} F_{1}}{\partial u^{2}} \frac{\partial^{2} F_{2}}{\partial u^{2}}+\frac{\partial^{2} F_{1}}{\partial v^{2}} \frac{\partial^{2} F_{2}}{\partial v^{2}}\right]\left.\right|_{u=0, v=0},
\end{gathered}
$$

by some tedious calculations, we see that the sign of $\sigma$ is determined by $\xi$, where

$$
\begin{aligned}
\xi= & A^{2} q_{1}\left(2 d \rho q_{1}+\mu p^{2}\left(2 d p-\mu \rho-4 p^{2}\right)\right) \\
& +p^{2}\left(p^{3}(\mu+d)(\mu+p)^{2}-3 q_{2} \rho A^{2} \omega^{2}\right) .
\end{aligned}
$$

By the results in [14], the direction of the Hopf bifurcation is determined by the sign of $\sigma$. Therefore, we have the following result.

Theorem 6. Suppose one condition of (c) in Theorem (6) holds and $r_{0}=r_{1}$. If $\xi \neq 0$, then a curve of periodic solutions bifurcates from the endemic equilibrium $E_{2}$ such that 
(i) for $\xi<0$, system (3) undergoes a supercritical Hopf bifurcation;

(ii) for $\xi>0$, system (3) undergoes a subcritical Hopf bifurcation.

Remark 7. Theorems 5 and 6 imply the occurrence of the Allee effect because endemic equilibrium $E_{2}$ and the diseasefree equilibrium can be stable at the same time, or a stable limit cycle and the disease-free equilibrium can be stable at the same time.

3.2. Bogdanov-Takens Bifurcations. The purpose of this subsection is to study the Bogdanov-Takens bifurcation of (3) when there is a unique degenerate positive equilibrium. Assume that

(H1)

$$
\begin{aligned}
& \text { (1) } a>0, R_{0}>1+\sqrt{3 \beta a / d} \text { and } 2 a_{1}^{3 / 2}=a_{2} \\
& \text { (2) } a=0 \text { and } R_{0}=1+\sqrt[3]{27 \beta^{2} b / 4 d^{2}} \\
& \text { (3) } a<0 \text { and } 2 a_{1}^{3 / 2}=a_{2} \text {. }
\end{aligned}
$$

Then system (3) admits a unique positive equilibrium $\left(S^{*}, I^{*}\right)$ if one of $(\mathrm{H} 1)$ is satisfied.

The Jacobian matrix of (3) at this point is

$$
J=\left(\begin{array}{cc}
-d-\frac{\beta I^{* 3}}{b+a I^{*}+I^{* 2}} & -\mu-\frac{\mu\left(2 b+a I^{*}\right)}{b+a I^{*}+I^{* 2}} \\
\frac{\beta I^{* 3}}{b+a I^{*}+I^{* 2}} & \frac{\mu\left(2 b+a I^{*}\right)}{b+a I^{*}+I^{* 2}}
\end{array}\right) \text {. }
$$

Since we are interested in codimension 2 bifurcations, we assume further

(H2) $r_{0}=r_{1}$

By (15), we have

$$
\operatorname{det}(J)=\frac{\beta \mu I^{* 3}-d a \mu I^{*}-2 d b \mu}{b+a I^{*}+I^{* 2}}=0 .
$$

Furthermore, (H2) implies that

$$
\operatorname{tr}(J)=\frac{-\beta A I^{* 2}+a \mu^{2} I^{*}+2 b \mu^{2}}{\mu\left(b+a I^{*}+I^{* 2}\right)}=0 .
$$

Thus, (H1) and (H2) imply that the Jacobian matrix has a zero eigenvalue with multiplicity 2 . This suggests that (3) may admit a Bogdanov-Takens bifurcation. The next theorem will confirm this.

Theorem 8. Suppose that (H1) and (H2) hold. Then the equilibrium $\left(S^{*}, I^{*}\right)$ of (3) is a cusp of codimension 2 ; that is, it is a Bogdanov-Takens singularity.

Proof. In order to translate the interior equilibrium $\left(S^{*}, I^{*}\right)$ to the origin, we set $x=S-S^{*}, y=I-I^{*}$. Expanding the right-hand side of the system (3) in a Taylor series about the origin, we obtain

$$
\begin{gathered}
\frac{d x}{d t}=a_{11} x+a_{12} y+a_{21} x y+a_{22} y^{2}+P_{1}(x, y), \\
\frac{d y}{d t}=-\frac{a_{11}^{2}}{a_{12}} x-a_{11} y-a_{21} x y-a_{22} y^{2}+P_{2}(x, y),
\end{gathered}
$$

where $P_{i}(x, y)$ is a smooth function in $(x, y)$ at least of order three and

$$
\begin{gathered}
a_{11}=-d-\frac{\beta I^{* 3}}{b+a I^{*}+I^{* 2}}<0, \\
a_{12}=-\mu-\frac{\mu\left(2 b+a I^{*}\right)}{b+a I^{*}+I_{2}^{* 2}}<0, \\
a_{21}=\frac{-\beta I^{* 2}\left(3 b+2 a I^{*}+I^{* 2}\right)}{\left(b+a I^{*}+I^{* 2}\right)^{2}}<0, \\
a_{22}=\frac{-2 \beta S^{*} I^{*}\left[3 b^{2}+3 a b I^{*}+\left(a^{2}-b\right) I^{* 2}\right]}{\left(b+a I^{*}+I^{* 2}\right)^{3}} .
\end{gathered}
$$

Set $X=x, Y=a_{11} x+a_{12} y$. Then (32) is transformed into

$$
\begin{aligned}
& \frac{d X}{d t}=Y+c_{1} X^{2}+c_{2} X Y+c_{3} Y^{2}+Q_{1}(X, Y), \\
& \frac{d Y}{d t}=-d_{1} X^{2}+d_{2} X Y+d_{3} Y^{2}+Q_{2}(X, Y),
\end{aligned}
$$

where $Q_{i}$ are smooth functions in $(X, Y)$ at least of order three and

$$
\begin{gathered}
c_{1}=\frac{a_{11}\left(a_{11} a_{22}-a_{12} a_{21}\right)}{a_{12}^{2}}, \\
d_{1}=\frac{a_{11}\left(a_{12} a_{21}-a_{11} a_{22}\right)\left(a_{11}-a_{12}\right)}{a_{12}^{2}}, \\
c_{2}=\frac{a_{12} a_{21}-2 a_{11} a_{22}}{a_{12}^{2}}, \\
d_{2}=\frac{\left(a_{12} a_{21}-2 a_{11} a_{22}\right)\left(a_{11}-a_{12}\right)}{a_{12}^{2}} \\
c_{3}=\frac{a_{22}}{a_{12}^{2}} \\
d_{3}=\frac{a_{22}\left(a_{11}-a_{12}\right)}{a_{12}^{2}} .
\end{gathered}
$$

Change the variables one more time by letting $X=X, P=$ $Y+c_{3} Y^{2}$; we have

$$
\begin{gathered}
\frac{d X}{d t}=P+c_{1} X^{2}+c_{2} X Y+Q_{3}(X, P), \\
\frac{d P}{d t}=-d_{1} X^{2}+d_{2} X P+d_{3} P^{2}+Q_{4}(X, P) .
\end{gathered}
$$


Let $X=X, Z=P-d_{3} X P$. Then system (36) becomes

$$
\begin{gathered}
\frac{d X}{d t}=Z+c_{1} X^{2}+\left(c_{2}+d_{3}\right) X Z+Q_{5}(X, Z), \\
\frac{d Z}{d t}=-d_{1} X^{2}+d_{2} X Z+Q_{6}(X, Z) .
\end{gathered}
$$

In order to obtain the canonical normal forms, we perform the transformation of variables by

$$
u=X-\frac{c_{2}+d_{3}}{2} X^{2}, \quad v=Z+c_{1} X^{2}
$$

Then, we obtain

$$
\begin{gathered}
\frac{d u}{d t}=v+R_{1}(u, v), \\
\frac{d v}{d t}=-d_{1} u^{2}+\left(d_{2}+2 c_{1}\right) u v+R_{2}(u, v),
\end{gathered}
$$

where $R_{i}$ are smooth functions in $(u, v)$ at least of the third order.

Note that $d_{1}>0$ and

$$
d_{2}+2 c_{1}=\frac{-a_{11} a_{21}-a_{21} a_{12}+2 a_{22} a_{11}}{a_{12}} .
$$

In addition, by (30) and (31), it is obtained that

$$
\begin{gathered}
a_{11}=-\frac{A}{S^{*}}, \quad a_{12}=-\mu-\frac{A}{S^{*}}, \\
a_{21}=\frac{\mu I^{*}}{S^{*}}, \quad a_{22}=\frac{A}{S^{*}}, \\
\frac{A^{2}}{S^{* 2}}=\frac{\mu I^{*}}{S^{*}}\left(\mu+\frac{A}{S^{*}}\right) .
\end{gathered}
$$

So

$$
\begin{aligned}
d_{2}+2 c_{1} & =\frac{-a_{11} a_{21}-a_{21} a_{12}+2 a_{22} a_{11}}{a_{12}} \\
& =\frac{1}{a_{12}} \frac{A\left(\mu I^{*}-A\right)}{S^{* 2}}>0 .
\end{aligned}
$$

It follows that (3) admits that a Bogdanov-Takens bifurcation from $[15,16]$ or [17].

\section{Simulations and Conclusions}

In the following, we use numerical simulations, based upon the MatCont package [18], to reveal how parameters $a$ induce bifurcations and limit cycles in system (3). Firstly, by fixing $A=2, d=0.1, \beta=0.8, b=2.4, \epsilon=0.6, \gamma=0.2$, we plot a $2 \mathrm{D}$-plot of variable $I$ versus parameter $a$ shown in Figure 3. We find a Hopf bifurcation at $a=-0.090429$, a limit point (fold) bifurcation at $a=2.378982$. The Lyapunov coefficient is $1.68171 \times 10^{-2}$, which means that the periodic orbits are unstable. Furthermore, $a$ is fixed -0.13 ; we observe the orbits of system (3) is how to vary with $t$. From Figure 4, we can find

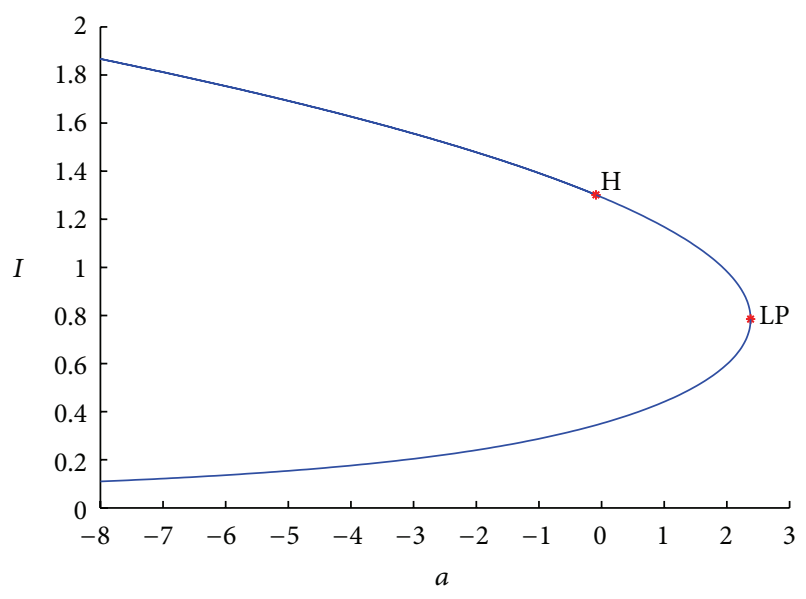

Figure 3: Bifurcation curves in $(a, I)$ plane by fixed $A=2, d=$ $0.1, \beta=0.8, b=2.4, \epsilon=0.6, \gamma=0.2$, where $\mathrm{H}$ denotes the Hopf bifurcation, LP is the limit point (flod) bifurcation.

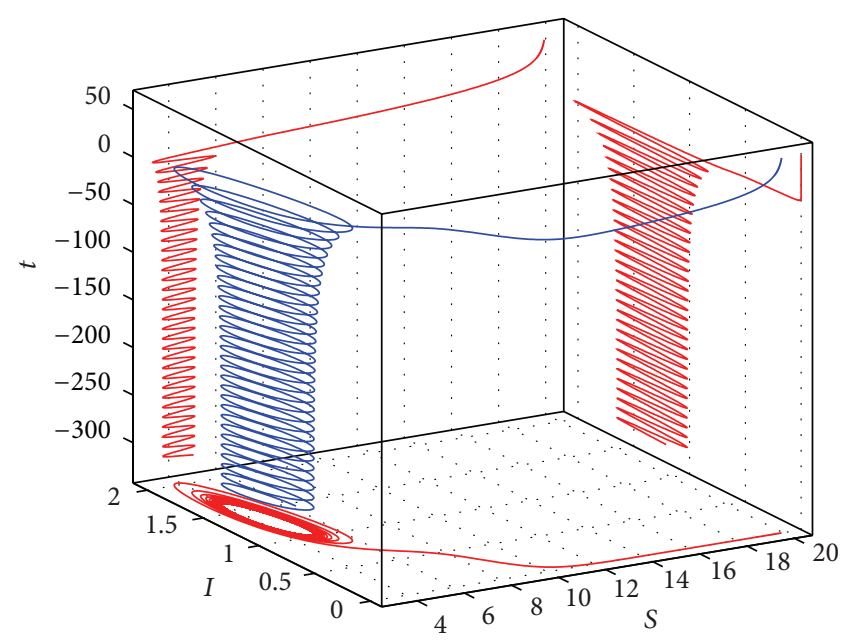

Figure 4: Phase trajectory in system (3) by fixed $A=2, d=0.1, \beta=$ $0.8, b=2.4, \epsilon=0.6, \gamma=0.2, a=-0.13$.

that the periodic orbits will occur, but the disease will die out when $t \rightarrow+\infty$, though there exist the positive equilibria for system (3). Furthermore, we take $R_{0}$ and $a$ as bifurcation parameters; from Figure 5, we can show that the system has no positive equilibrium when $R_{0}$ and $a$ lie in the left side of red curve and two endemic equilibria when they are in the right side of red curve. If parameters $R_{0}$ and $a$ are between red and green curves, we find that system will undergo Hopf bifurcation.

In the paper, we built a model with contact transmission function and obtained the dynamical behaviors. From the analysis, we find that the threshold value of disease spreading will be larger. It means that it is an effective intervention policy adopted for disease spreading. For the disease-free equilibrium is always locally stable and when a positive equilibrium exist and is stable, we can control the disease as long as we let the initial values be in the certain range by intervention policy. If the positive equilibrium is unstable, the 


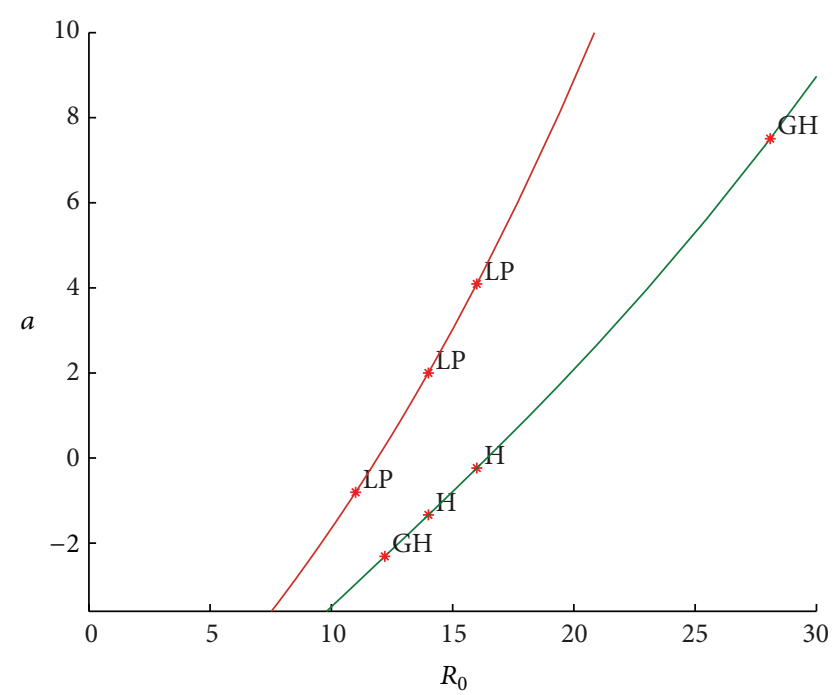

FIGURE 5: Bifurcation figure when $R_{0}$ and $a$ are taken as bifurcation parameters in system (3) by fixed $A=2, d=0.1, \beta=0.8, b=2.4, \epsilon=$ $0.6, \gamma=0.2$.

disease will die out. This will provide a theoretical basis for the prevention and control of disease.

\section{Conflict of Interests}

The authors declare that there is no conflict of interests regarding the publication of this paper.

\section{Acknowledgments}

This work is supported by the National Science Foundation of China (11201434, 11271369) and Shanxi Scholarship Council of China (2013-087).

\section{References}

[1] V. Capasso and G. Serio, "A generalization of the KermackMcKendrick deterministic epidemic model," Mathematical Biosciences, vol. 42, no. 1-2, pp. 43-61, 1978.

[2] S. Ruan and W. Wang, "Dynamical behavior of an epidemic model with a nonlinear incidence rate," Journal of Differential Equations, vol. 188, no. 1, pp. 135-163, 2003.

[3] Y. Tang, D. Huang, S. Ruan, and W. Zhang, "Coexistence of limit cycles and homoclinic loops in a SIRS model with a nonlinear incidence rate," SIAM Journal on Applied Mathematics, vol. 69, no. 2, pp. 621-639, 2008.

[4] D. Xiao and H. Zhu, "Multiple focus and Hopf bifurcations in a predator-prey system with nonmonotonic functional response," SIAM Journal on Applied Mathematics, vol. 66, no. 3, pp. 802819, 2006.

[5] W. Wang, "Epidemic models with nonlinear infection forces," Mathematical Biosciences and Engineering, vol. 3, no. 1, pp. 267279, 2006.

[6] D. Xiao and S. Ruan, "Global analysis of an epidemic model with nonmonotone incidence rate," Mathematical Biosciences, vol. 208, no. 2, pp. 419-429, 2007.
[7] A. B. Gumel and S. M. Moghadas, "A qualitative study of a vaccination model with non-linear incidence," Applied Mathematics and Computation, vol. 143, no. 2-3, pp. 409-419, 2003.

[8] W. M. Liu, H. W. Hethcote, and S. A. Levin, "Dynamical behavior of epidemiological models with nonlinear incidence rates," Journal of Mathematical Biology, vol. 25, no. 4, pp. 359380, 1987.

[9] W. M. Liu, S. A. Levin, and Y. Iwasa, "Influence of nonlinear incidence rates upon the behavior of SIRS epidemiological models," Journal of Mathematical Biology, vol. 23, no. 2, pp. 187204, 1986.

[10] R. R. Regoes, D. Ebert, and S. Bonhoeffer, "Dose-dependent infection rates of parasites produce the Allee effect in epidemiology," Proceedings of the Royal Society B, vol. 269, no. 1488, pp. 271-279, 2002.

[11] G. Li and W. Wang, "Bifurcation analysis of an epidemic model with nonlinear incidence," Applied Mathematics and Computation, vol. 214, no. 2, pp. 411-423, 2009.

[12] R. M. Anderson and R. M. May, "Population biology of infectious diseases: Part I," Nature, vol. 280, no. 5721, pp. 361367, 1979.

[13] R. M. May and R. M. Anderson, "Population biology of infectious diseases: Part II," Nature, vol. 280, no. 5722, pp. 455461, 1979.

[14] L. Perko, Differential Equations and Dynamical Systems, vol. 7, Springer, New York, NY, USA, 2nd edition, 1996.

[15] R. Bogdanov, "Bifurcations of a limit cycle for a family of vector fields on the plan," Selecta Mathematica, vol. 1, pp. 373-388, 1981.

[16] R. Bogdanov, "Versal deformations of a singular point on the plan in the case of zero eigenvalues," Selecta Mathematica, vol. 1, pp. 389-421, 1981.

[17] F. Takens, "Forced oscillations and bifurcations," in Applications of Global Analysis I, pp. 1-59, Rijksuniversitat Utrecht, 1974.

[18] A. Dhooge, W. Govaerts, and Y. A. Kuznetsov, "Limit cycles and their bifucations in MatCont," http://www.matcont.ugent.be/ Tutorial2.pdf. 


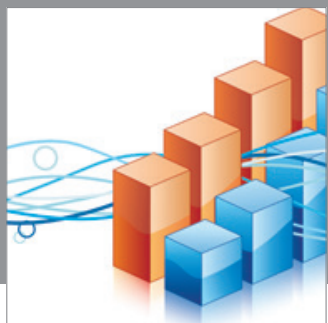

Advances in

Operations Research

mansans

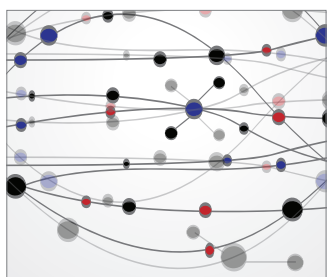

The Scientific World Journal
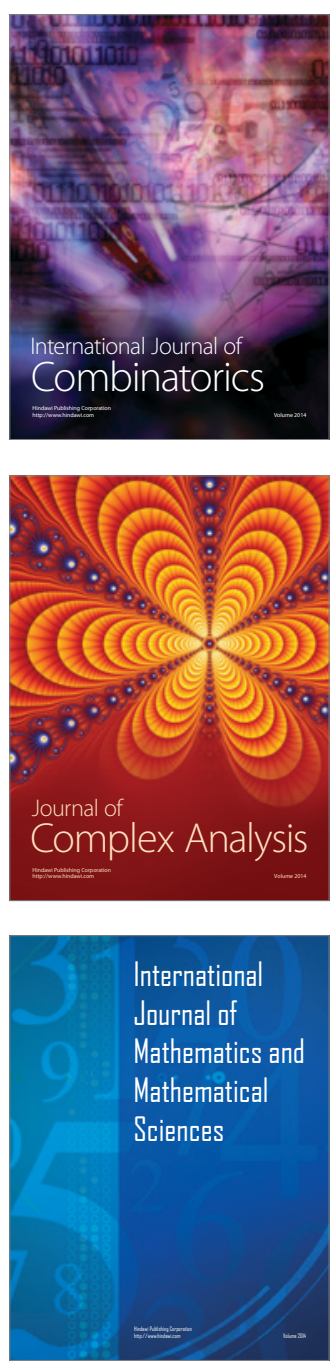
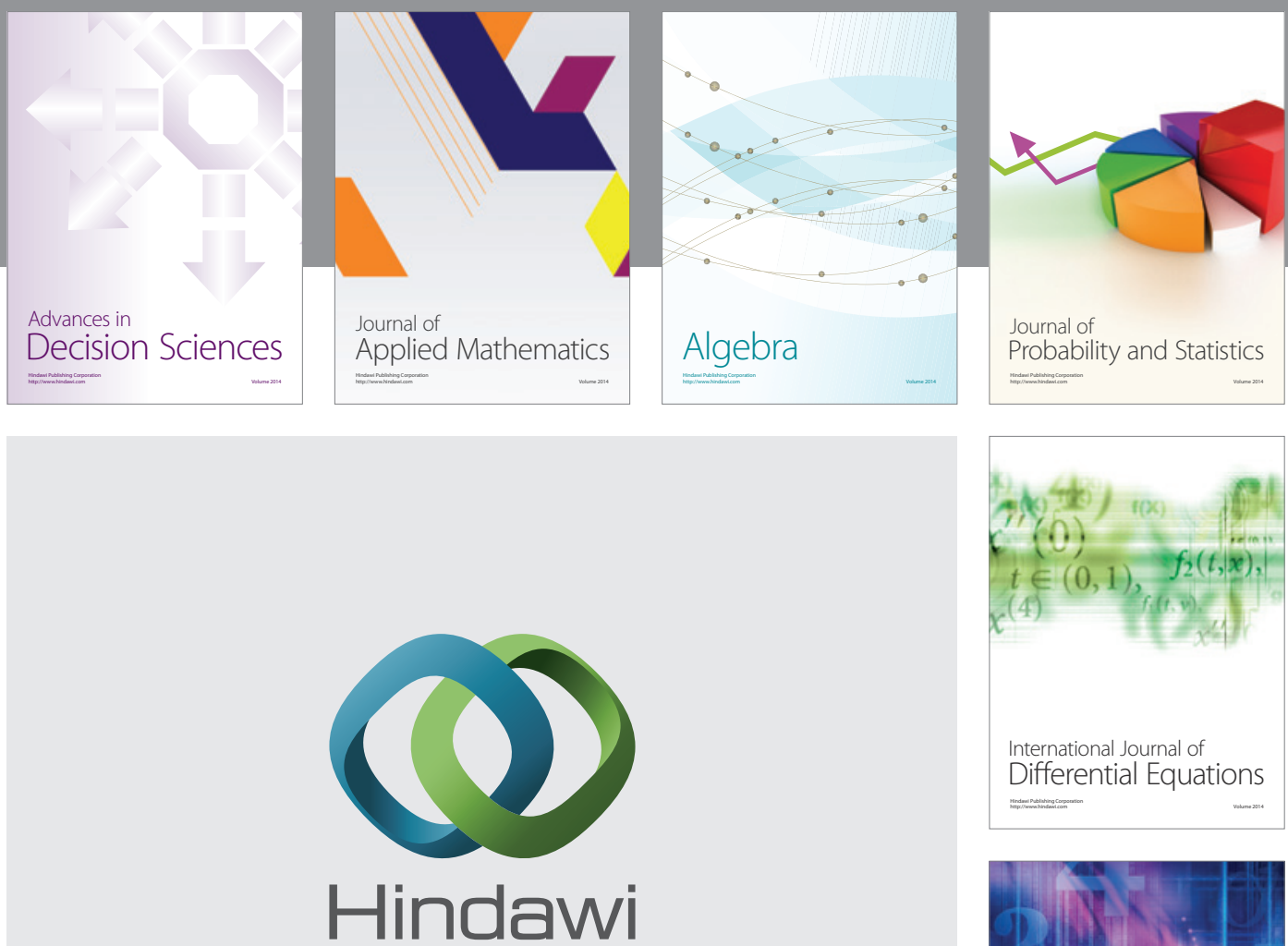

Submit your manuscripts at http://www.hindawi.com
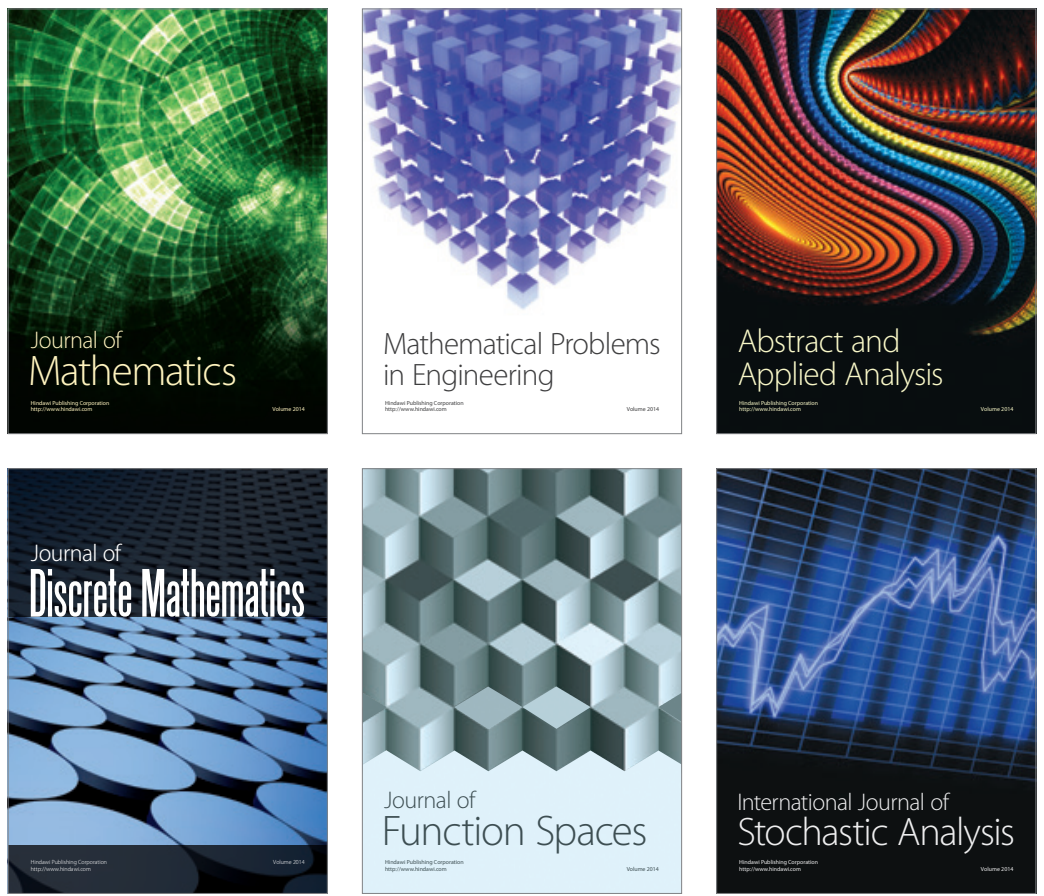

Journal of

Function Spaces

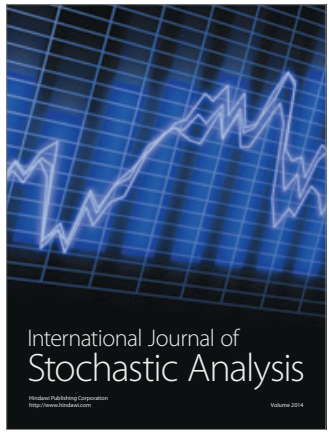

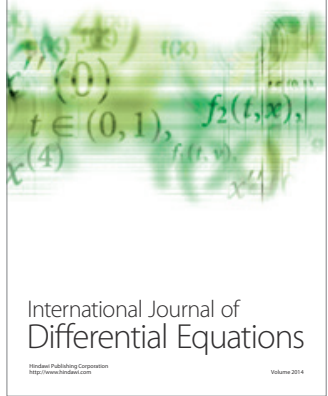
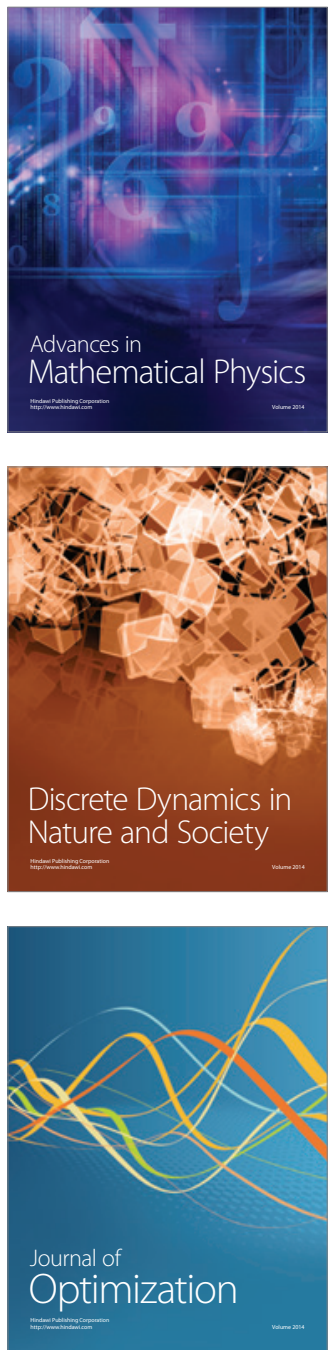